\title{
Cardiopulmonale Wiederbelebung in Zeiten der COVID-19 Pandemie
}

\author{
Wolfgang Lederer
}

Angenommen: 21. Januar 2021 / Online publiziert: 9. Februar 2021

(C) Springer-Verlag GmbH Deutschland, ein Teil von Springer Nature 2021

\section{Einleitung}

Seit einem Jahr ist die weltweite Ausbreitung einer Infektion mit SARS-CoV-2 (severe acute respiratory syndrome - associated corona virus 2) das bestimmende Thema in den meisten Ländern (van Damme et al. 2020). Die anhaltend große Aufmerksamkeit fokusiert besonders auf gesundheitliche und sozioökonomische Belastungen und geht mit enormen Einschränkungen für das Leben der Menschen einher. Die Angst vor Ansteckung mit der potenziell fatal verlaufenden Krankheit durch Kontakt mit bereits infizierten Personen wird durch die mediale Aufbereitung von täglich aktualisierten Infektions- und Sterbestatistiken auf hohem Niveau gehalten. Im Schatten rigoroser Lockdown Szenarien gedeihen Verunsicherung, Diskriminierung und Ängste. Entsprechend groß ist auch die Verzweiflung, wenn man beim plötzlichen Herzkreislaufstillstand eines Angehörigen sich im Konflikt zwischen unterlassener Hilfeleistung und möglicher Selbstgefährdung vollkommen allein gelassen fühlt.

\section{Cardiopulmonale Reanimation während der COVID-19 Pandemie}

In einer Studie, die in Paris im Frühjahr 2020 durchgeführt wurde, konnte festgestellt werden, dass zu Beginn der COVID-19 Krise Ersthelfer-Maßnahmen im Vergleich zu den Jahren vor der Pandemie deutlich seltener vorgenommen wurden (Marijon et al. 2020). Zusätzlich waren auch die Intervalle bis zum Eintreffen der qualifizierten Helfer signifikant länger. Entsprechend schlecht waren die Reanimationsergebnisse

Univ.-Prof. Dr. W. Lederer ( $\triangle)$

Universitätsklinik für Anästhesie und Intensivmedizin, Medizinische Universität Innsbruck,

Anichstr. 35, 6020 Innsbruck, Österreich

E-Mail: wolfgang.lederer@i-med.ac.at 
in dieser Untersuchung, obwohl in weniger als $10 \%$ der Patienten eine Infektion mit SARS-CoV-2 nachgewiesen wurde.

Die Autoren schließen daraus, dass in Zeiten der COVID-19 Pandemie die Bereitschaft zur Hilfeleistung durch Ersthelfer deutlich zurückgegangen ist (Marijon et al. 2020). Das wäre eine Erklärung für den Qualitätsverlust in der prähospitalen CPR (cardiopulmonalen Reanimation) und die davon abhängende Chance auf Überleben (Baldi et al. 2020). Reanimation wird als aerosolbildende Tätigkeit eingestuft, mit einem Gefährdungpotential für über Atemluft übertragbare Erkrankungen (Klompas et al. 2020). Die Beatmung gehört seit jeher zu den kritischen Maßnahmen der Wiederbelebung. Einerseits, weil die Mund-zu-Mund Beatmung von fremden Personen für die Ersthelfer immer eine Überwindung von Ekel und Abscheu mitbringt. Andererseits, weil das Gehirn nach Herzkreislaufstillstand eine sehr geringe Hypoxietoleranz hat und bezogen auf seine Masse im Vergleich zu anderen Organen einen um ein Vielfaches höheren Sauerstoffbedarf hat (Kissoon et al. 1990). Speziell bei Kindern hat frühe und effiziente Beatmung einen enormen Einfluss auf den erreichbaren Reanimationserfolg, da Kreislaufstillstände bei Kindern hauptsächlich durch Sauerstoffmangel verursacht werden (Meert et al. 2016).

Wegen der eingeschränkten Versorgungsmöglichkeiten während der COVID-19 Pandemie empfehlen aktualisierte internationale Leitlinien der Reanimation für Ersthelfer die Verwendung eines AED (automatisierter externer Defibrillator) und die alleinige Durchführung von Kompressionen ohne Beatmung (Nolan et al. 2020). Diese pragmatische Vorgangsweise basiert auf der Beobachtung, dass in prähospitalen Studien bei Erwachsenen mit Kreislaufstillstand aus kardialer Genese in den ersten zehn Minuten nach Stillstand kein signifikanter Unterschied im Überleben nach erfolgreichen Reanimationen mit und ohne Beatmung feststellbar war. Daraus wurde geschlossen, dass zur Überbrückung bis zum Eintreffen von professionellen Helfern eine kurzzeitige Perfusion mit wenig oxygeniertem Blut besser ist als gar keine.

Leider wird durch die COVID-19 Pandemie auch die Ausbildung von Ersthelfern in Wiederbelebung beeinträchtigt (Baldi et al. 2020). Mund-zu-Mund Beatmung kann effektiv nur am Phantom gelernt werden. Trotz häufiger Oberfächendesinfektion und Wechsel der Beatmungslungen am Phantom kann das Risiko einer Infektion nicht absolut ausgeschlossen werden. Der Verzicht auf manuelles Training am Phantom hat nachhaltige Auswirkungen auf die Qualität der Ausbildung und die davon abhängige Qualität der Ersthelfer-CPR (Baldi et al. 2020).

Bedeutet das nun, dass man beim prähospitalen Herzkreislaufstillstand als Folge der COVID-19 Pandemie keine Beatmung mehr durchführt oder sogar gänzlich auf CPR verzichtet (Mahase und Kmietowicz 2020)?

\section{Ethische Prinzipien}

Idealerweise sollten CPR-Maßnahmen nur bei Aussicht auf nachhaltigen Erfolg bei plötzlich unerwartetem Herzkreislaufstillstand mit potenziell reversiblen Ursachen durchgeführt werden. Zusätzlich zu den Ersthelfer-Maßnahmen braucht es qualifizierte Helfer, die innerhalb kurzer Zeit mit den erweiterten Maßnahmen der Reani- 
mation beginnen können. Kommt es zum Wiedereintritt eines spontanen Kreislaufes, muss auch noch ein freier Überwachungsplatz mit Beatmungs- und Intensivtherapiemöglichkeiten in einem Krankenhaus organisiert werden (Lederer 2016, S. 221). Gerade zu Beginn der COVID-19 Pandemie waren sowohl die Verfügbarkeit von qualifizierten Helfern, als auch die Anzahl von mit Intensivpersonal ausreichend betreuten Überwachungsplätzen im Krankenhaus sehr eingeschränkt.

Medizinische Behandlungen sind ethischen Grundlagen verpflichtet und dürfen nicht nach unsachlichen Kriterien entschieden werden. Es gilt das Optimierungsgebot. Daraus resultiert die Pflicht, Risiken zu minimieren und Diskriminierung zu vermeiden (Bioethikkommission 2020, S. 5). Auch wenn zahlreiche medizinischfachliche, rechtliche und sozioökonomische Kriterien während einer Pandemie zu berücksichtigen sind, dürfen ethische Prinzipien (Beauchamp and Childress 2008) nicht grundsätzlich in Frage gestellt werden.

Das betrifft insbesondere die Prinzipien der Schadensvermeidung und der Gerechtigkeit.

Schadensvermeidung bezieht sich nicht nur auf die Beendigung des ursächlichen Schadensereignisses und das Vermeiden weiterer Schäden durch die Versorgung für den Patienten. Es schließt auch mit ein, dass Helfer und Helferinnen durch Gefahren und Belastungen am Notfallort nicht zu Schaden kommen dürfen. Quälende Selbstvorwürfe aus Handlungen (,Das hätte ich nicht mehr tun sollen“) oder Unterlassungen (,Das hätte ich doch machen sollen“) können zu nachhaltigen Belastungen der Helfer führen (Lederer 2016, S. 220).

Die emotionale Belastung der Helfer am Notfallort verändert auch deren Einschätzung von Risiken und die Bereitschaft Risiken zu tolerieren. So werden besonders bei Kindern und jungen Patienten mit Herzkreislaufstillstand die CPR-Maßnahmen durch Ersthelfer und professionelle Helfer intensiver und länger durchgeführt als bei Erwachsenen (Iserson und Stocking 1993).

\section{Das aktuelle Dilemma}

Während der COVID-19 Pandemie wird die Verantwortungsbereitschaft von Ersthelfern maximal gefordert. Die Risikowahrscheinlichkleit vor Ort kann nur sehr schwer abgeschätzt werden. Das Fehlen von typischen Symptomen, die für eine Erkankung mit SARS-CoV-2 sprechen würden, kann für den Auschluss einer Infektion nicht herangezogen werden, da ja bis zu $90 \%$ der Infektionen asymptomatisch oder mild verlaufen (Petersen und Phillips 2020). Selbst ein aktuell negativer Screening-Test schließt eine Infektion nicht hundertprozentig aus.

Seit der COVID-19 Pandemie empfehlen die meisten Good Practice Statements und Consensus On Recommendations von Internationalen Gesellschaften, dass Ersthelfer bei der Reanimation nur Kompressionen durchführen (compression-only CPR) und mit einem AED defibrillieren (public-access defibrillation), wenn es angezeigt ist (Nolan et al. 2020). Das führt aber zu einer deutlichen Verschlechterung der Überlebenschancen von Personen mit plötzlichem Herzkreislaufstillstand aus respiratorischer Ursache. Dieser ungleiche Zugang zu CPR Maßnahmen widerspricht dem Prinzip der Gerechtigkeit. 
Auch wenn keine Beatmung durchgeführt wird, besteht das Risiko einer Infektionsübertragung für die Ersthelfer. Selbst mit Schutzanzügen, Masken und Handschuhen gibt es keine absolute Sicherheit vor Infektion (Seto et al. 2003). Die aktuellen Empfehlungen nehmen also trotz der Einschränkungen einen möglichen Schaden für die Ersthelfer in Kauf. Auch gilt für die chest compression-only CPR durch Ersthelfer, dass rasch eintreffende qualifizierte Helfer so früh wie möglich mit den erweiterten Maßnahmen der Wiederbelebung einschließlich der Beatmung beginnen, um die Chance für ein gutes Outcome zu wahren. Deshalb beinhalten die Empfehlungen der aktualisierten Internationalen Leitlinien der Reanimation während der COVID-19 Pandemie den Zusatz, dass Ersthelfer, die in der Beatmung geübt sind und sich die Durchführung zumuten, bei der Reanimation von Kindern eine Mundzu-Mund Beatmung zusätzlich zur Herzdruckmassage durchführen können (Nolan et al. 2020).

Um ein unverhältmäßiges Risiko für das Reanimationsteam zu vermeiden ist es daher wichtig, die Sicherheitsmaßnahmen weiter zu optimieren (Bioethikkommission 2020, S. 5). Die im Vergleich zur alljährlich auftretenden Influenza-Epidemie erhöhte Letalität durch SARS-CoV-2 hat zu einem kompletten Umdenken in der Risikobeurteilung für Helfer am Notfallort geführt. Bis zum Beginn der COVID19 Pandemie wurden Beatmungstücher und Beatmungsmasken als ausreichender Schutz vor Infektion eingestuft (Baldi et al. 2020; Ogawa et al. 2011). Beatmungsmasken sind nicht effizienter als Mund-zu-Mund Beatmung, aber sie erhöhen die Bereitschaft, eine Beatmung während der CPR durchzuführen. Beatmungstücher mit eingebautem Ventil verringern die Virus Exposition. Eine neue Maßnahme wäre die Abdeckung des Patienten während der Reanimation mit einer $120 \times 120 \mathrm{~cm}$ Polyvinylchlorid Folie. Damit könnte der Kontakt mit Ausatemluft, Erbrochenem, Blut und Körpersekreten des Patienten vermindert werden. Speziell Aerosole können durch Adhäsion und Drainage von den Helfern abgeschirmt werden (Lederer et al. 2020). Trotz der Abdeckung des gesamten Oberkörpers ist eine Beatmung über eine zentrale, dicht eingebaute Beatmungsmaske mit integriertem Beatmungsfilter möglich. Das verbessert nicht nur die Qualität der CPR, sondern erhöht auch den Schutz der Ersthelfer und die Bereitschaft, die Wiederbelebung mit Beatmung durchzuführen.

\section{Fazit}

Um das Infektionsrisiko für die Helfer zu vermindern und die eingeschränkte Qualität der CPR während der COVID-19 Pandemie wieder zu verbessern, braucht es verschiedene Optimierungsmaßnahmen. Ein Ansatz wäre die Abdeckung des Patienten mit einer Polyvinylchlorid Folie, die eine zentral eingebaute Beatmungmaske mit integriertem Beatmungsfilter hat. Damit kann die Kontamination mit Aerosol, Erbrochenem, Blut und Körpersekreten des Patienten vermindert werden. Öffentlich zugängliche AED Standorte sollten zusätzlich mit Einmalhandschuhen und einer Folie mit Beatmungmaske und integriertem Filter ausgerüstet werden. 


\section{Einhaltung ethischer Richtlinien}

Interessenkonflikt W. Lederer gibt an, dass kein Interessenkonflikt besteht.

Ethische Standards Für diesen Beitrag wurden vom Autor keine Studien an Menschen oder Tieren durchgeführt. Für die aufgeführten Studien gelten die jeweils dort angegebenen ethischen Richtlinien.

\section{Literatur}

Baldi E, Contri E, Savastano S, Cortegiani A (2020) The challenge of laypeople cardio-pulmonary resuscitation training during and after COVID-19 pandemic. Resuscitation 152:3-4

Beauchamp TL, Childress J (2008) Principles of biomedical ethics. Oxford University Press, Oxford

Bioethikkommission (2020) Zum Umgang mit knappen Resourcen in der Gesundheitsversorgung im Kontext der Covid-19-Pandemie. Bundeskanzleramt Österreich, Wien

van Damme W et al (2020) The COVID-19 pandemic: diverse contexts; different epidemics-how and why? BMJ Global Health 5(7):e3098. https://doi.org/10.1136/bmjgh-2020-003098

Iserson KV, Stocking C (1993) Standards and limits: emergency physicians' attitude toward prehospital resuscitation. Am J Emerg Med 11:592-594

Kissoon N, Dreyer J, Walia M (1990) Pediatric trauma: differences in pathophysiology, injury patterns and treatment compared with adult trauma. CMAJ 142(1):27-34

Klompas M, Baker MA, Rhee C (2020) Airborne transmission of SARS-CoV-2: theoretical considerations and available evidence. JAMA 324(5):441-442

Lederer W (2016) CPR-Entscheidungen in der prähospitalen Notfallmedizin. In: Neumayr A, Baubin M, Schinnerl A (Hrsg) Risikomanagement in der prähospitalen Notfallmedizin. Werkzeuge, Maßnahmen, Methoden. Springer, Berlin Heidelberg

Lederer W, Isser M, Wiedermann FJ (2020) COVID-19 pandemic: ventilation during basic life support. https://professional.heart.org/professional/GuidelinesStatements/Correspondence/UCM_320919_ Correspondence.jsp. Zugegriffen: 13. Jan. 2021 (Correspondence AHA website: President's Pages)

Mahase E, Kmietowicz Z (2020) Covid-19: doctors are told not to perform CPR on patients in cardiac arrest. BMJ. https://doi.org/10.1136/bmj.m1282

Marijon E et al (2020) Out-of-hospital cardiac arrest during the COVID-19 pandemic in Paris, France: a population-based, observational study. Lancet Public Health. https://doi.org/10.1016/S24682667(20)30117-1

Meert KL et al for the Therapeutic Hypothermia after Pediatric Cardiac Arrest (THAPCA) Trial Investigators (2016) Pediatric out-of-hospital cardiac arrest characteristics and their association with survival and neurobehavioral outcome. Pediatr Crit Care Med 17(12): e543-e550

Nolan JP et al (2020) European Resuscitation Council COVID-19 guidelines executive summary. Resuscitation 153:45-55

Ogawa T, Akahane M, Koike S, Tanabe S, Mizoguchi T, Imamura T (2011) Outcomes of chest compression only CPR versus conventional CPR conducted by lay people in patients with out of hospital cardiopulmonary arrest witnessed by bystanders: nationwide population based observational study. BMJ 342:c7106

Petersen I, Phillips A (2020) Three quarters of people with SARS-CoV-2 infection are asymptomatic: analysis of English Household Survey. Clin Epidemiol 12:1039-1043

Seto WH et al (2003) Advisors of Expert SARS group of Hospital Authority. Effectiveness of precautions against droplets and contact in prevention of nosocomial transmission of severe acute respiratory syndrome (SARS). Lancet 361(9368):1519-1520 